Induced Granulation Tissue but not Artificial Dermis Enhances

Early Host-Graft Interactions in Full-Thickness Burn Wounds

\title{
Lagus, Heli
}

2018-04

Lagus , H , Kankuri , E , Nuutila , K, Juteau , S, Sarlomo-Rikala , M \& Vuola , J 2018 ,'

Induced Granulation Tissue but not Artificial Dermis Enhances Early Host-Graft Interactions

in Full-Thickness Burn Wounds ' , World Journal of Surgery , vol. 42 , no. 4 , pp. 981-991 . https://doi.org/10.1007/s0

http://hdl.handle.net/10138/233887

https://doi.org/10.1007/s00268-017-4270-6

unspecified

publishedVersion

Downloaded from Helda, University of Helsinki institutional repository.

This is an electronic reprint of the original article.

This reprint may differ from the original in pagination and typographic detail.

Please cite the original version. 


\title{
Induced Granulation Tissue but not Artificial Dermis Enhances Early Host-Graft Interactions in Full-Thickness Burn Wounds
}

\author{
Heli Lagus ${ }^{1}$ Esko Kankuri ${ }^{2}$ Kristo Nuutila ${ }^{2,3}$ - Susanna Juteau ${ }^{4} \cdot$ \\ Maarit Sarlomo-Rikala ${ }^{4} \cdot$ Jyrki Vuola $^{5}$
}

Published online: 23 October 2017

(C) Société Internationale de Chirurgie 2017

\begin{abstract}
Background Cellular grafts used for skin repair require rapid integration with the host tissue to remain viable and especially to nourish the epidermal cells. Here, we evaluated the responses in the split-thickness skin grafts (STSGs) grafted on three differently treated wound beds: directly on excised wound bed (EX), on an artificial dermal template (DT) and on granulation tissue (GT) induced by cellulose sponge.

Methods In ten burn patients, after excision, a test area was divided into three sections: One transplanted with STSG instantaneously and two sections had a pre-treatment for 2 weeks with either DT or a cellulose sponge inducing granulation tissue formation and thereafter grafted with STSGs.

Results One week after grafting, the STSGs on GT demonstrated most endothelial $\mathrm{CD} 31^{+}$staining, largest average vessel diameters as well as most $\mathrm{CD}_{163^{+}}$staining of M2-like macrophages and most $\mathrm{MIB} 1^{+}$proliferating epidermal cells, suggesting an active regenerative environment. STSGs on DT had smallest vessel diameters and the least $\mathrm{CD} 63^{+}$macrophages. STSGs on EX had the least $\mathrm{CD} 31^{+}$cells and the least $\mathrm{MIB} 1^{+}$proliferating cells. After 3 months, this reactivity in STSGs had subsided, except increased dermal cell proliferation was observed in STSGs on EX.

Conclusions Results show that pre-treatment of wound bed and induction of granulation tissue formation can accelerate host-graft interaction by stimulating graft vasculature and inducing cell proliferation.
\end{abstract}

Electronic supplementary material The online version of this article (doi:10.1007/s00268-017-4270-6) contains supplementary material, which is available to authorized users.

\section{Heli Lagus}

heli.lagus@hus.fi

1 Department of Surgery, Lohja Hospital, The Hospital District of Helsinki and Uusimaa, Lohja, Finland

2 Department of Pharmacology, Faculty of Medicine, University of Helsinki, P.O. Box 63, 00014 Helsinki, Finland

3 Division of Plastic and Reconstructive Surgery, Brigham and Women's Hospital, Harvard Medical School, Boston, MA, USA

\section{Introduction}

Transplantation of split-thickness skin grafts (STSGs) is routinely used in the surgical treatment of both acute and chronic wounds. As a drawback, STSGs may not deliver

4 Department of Pathology, Haartman Institute, University of Helsinki and HUSLAB, P.O. Box 21, 00014 Helsinki, Finland

5 Department of Plastic Surgery, Helsinki University Hospital, P.O. Box 266, 00029 Helsinki, Finland 
enough dermal support for optimal host-graft interaction kinetics. Therefore, either temporary wound covers to induce formation of granulation tissue as the basis of new dermis or permanent dermal substitutes that introduce a novel dermis are often used. Temporary wound covers like cadaver skin, xenografts, allogeneic keratinocytes, synthetic membranes or different wound care products help to protect the wound [1] and to induce granulation tissue formation prior to grafting. Viscose cellulose sponges have been used as temporary wound covers to stimulate granulation tissue formation in, e.g., deep burns and traumatic injuries [2,3]. Permanent skin substitutes, on the other hand, replace either the dermis, epidermis or both, and the dermal substitutes offer a biodegradable support to assist STSG engraftment. These materials usually consist of matrix components with or without dermal and/or epidermal cells [4]. A widely used artificial dermal template (DT), Integra ${ }^{\circledR}$, made of type I collagen and chondroitin-6sulfate, has a matrix that facilitates the migration of fibroblasts and endothelial cells and therefore an ingrowth of new vessels into the material.

If, for example, the lack of available donor sites delays skin grafting, time should be used for priming the wound bed. Once grafting can be carried out, a primed wound bed can better promote the graft's take and support its integration. So far, mostly animal models have been used for studies investigating recipient site priming [5, 6]. These works have identified that wound bed vascularization is crucial for survival of epidermal cell layers and for re-establishing or maintaining a permanent barrier for fluids and microbes $[7,8]$. Both the quality and the quantity of neodermis or granulation tissue [9] are critical to wound healing. On the other hand, overgrowth of vascularized and collagenous granulation tissue has been associated with bad scar quality $[10,11]$. Thus, a well-primed wound bed with healthy granulation tissue or an active dermal substitute can crucially affect the outcome of skin replacement.

In this study, we investigated three wound bed priming strategies on their early effect on STSGs in patients with large full-thickness burns. The wound beds were (1) unprimed with an STSG grafted instantly after the excision, (2) primed with a permanent artificial dermal substitute or (3) primed with a viscose cellulose sponge to induce granulation tissue formation. Histological analyses were carried out from wound biopsies taken at 1-week and 3-month time points after transplantation of STSGs (Fig. 1). Graft vascularization was evaluated by CD31 endothelial cell staining and diameter of $\mathrm{CD} 31^{+}$vascular structures. The amount of M2-like regeneration-associated macrophages in STSGs was evaluated with CD163 staining. Our results provide the first insight into the graft response kinetics after host site priming in a clinically relevant setting [12].
Materials and methods

\section{Patients}

Ten adult patients (age range 19-58, mean $36.8 \pm 4.0$ years) with large deep burns consecutively admitted to Helsinki Burn Centre were included in the study. Total burned surface areas (TBSAs) ranged from 22 to $45 \%$ (mean $35.8 \pm 2.4 \%$ ). Patient demographics and the location of test areas are presented in Table 1. The study was approved by Research Ethics Committee of the Helsinki University Hospital (DNro 101/E6/ 2000), and informed consent was obtained from all subjects.

\section{Wounds and wound bed treatments}

Test areas were located within an anterior deep third-degree burn. Fascial wound excisions were performed 1-5 days after injury. Three adjacent areas (each $10 \times 5 \mathrm{~cm}$ ) were covered with: (1) a STSG instantly, (2) an artificial dermal template (Integra ${ }^{\circledR}$ ) and (3) a viscose cellulose sponge (Cellonex $^{\mathrm{TM}}$, Vivoxid Ltd, Finland) in random order. Wound beds of sections 2 and 3 were primed for 2 weeks before transplantation of STSGs. Figures 2, 3 show the study timeline, Fig. 2 illustrating the wound beds before and after treatments. The burn wounds surrounding the test sites were covered with meshed autografts. A monofilament polyamide woven dressing (Surfasoft ${ }^{\circledR}$, Mediprof, Bleiswijk, The Netherlands) and saline gauzes were used to cover the materials and the autografts. Topical antibiotics or silver was not used at test site or its immediate proximity.

All STSGs were harvested with a dermatome (Zimmer Inc, Warsaw, IN, USA). The 8/1000-in.-thick non-meshed grafts included epidermis, basement membrane and the superficial part of the dermis.

The DT used in this study has a biodegradable two-layer matrix with silicone providing protection against invasion of microbes and loss of fluids until replaced by STSG. The lower layer contains porous matrix of bovine tendon type I collagen

Table 1 Patient demographics, burn surface areas and test sites

\begin{tabular}{llll}
\hline Gender & Age & TBSA (\%) & Test location \\
\hline Male & 32 & 45 & Chest/abdomen \\
Male & 19 & 22 & Chest/abdomen \\
Male & 39 & 30 & Thigh \\
Female & 58 & 43 & Chest \\
Male & 54 & 40 & Chest \\
Male & 37 & 39 & Chest \\
Male & 40 & 33 & Chest \\
Male & 25 & 27 & Chest \\
Male & 41 & 42 & Chest/abdomen \\
Male & 23 & 37 & Thigh \\
\hline
\end{tabular}




\section{CONSORT 2010 Flow Diagram}

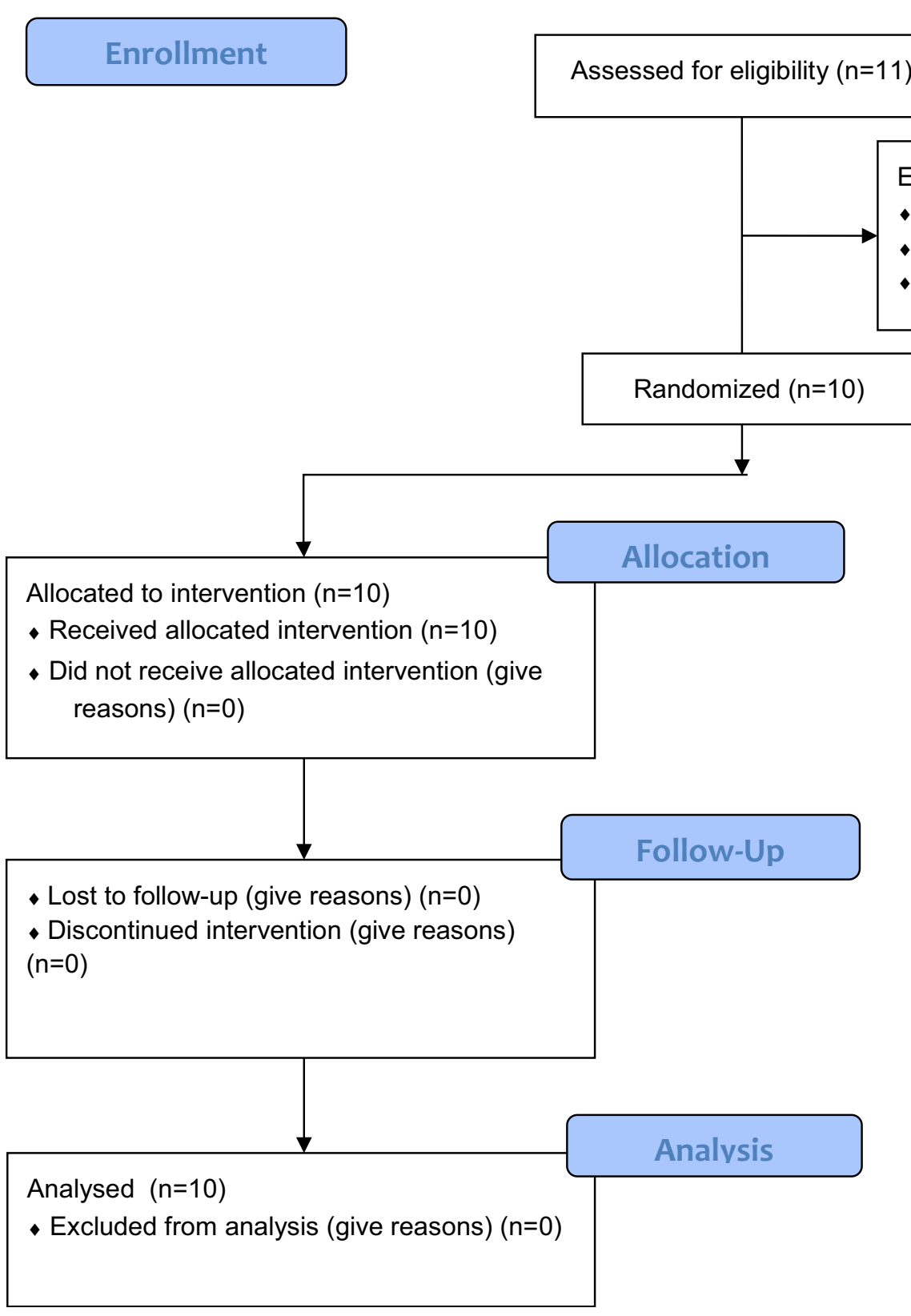

Fig. 1 CONSORT 2010 flow diagram

and chondroitin-6-sulfate (glycosaminoglycan) obtained from shark's cartilage. The porosity (mean pore diameter $30-120 \mu \mathrm{m})$ has been shown to facilitate the migration of inflammatory cells, fibroblasts and endothelial cells and thus promotes the ingrowth of new blood vessels into matrix [13].

The temporary wound cover used in this study was a sponge-like viscose cellulose matrix supported by cotton fibers. The viscose cellulose contains micropores with a diameter $1-10 \mu \mathrm{m}$ and macropores with a diameter $100-1000 \mu \mathrm{m}$. The rate of cell invasion and the formation of new tissue have been stated to be rapid in cellulose sponge [14].

Tissue samples, immunohistochemical stainings and analyses

Three-mm punch biopsies were taken as detailed in Fig. 4. Samples were fixed in $10 \%$ buffered formalin and embedded 

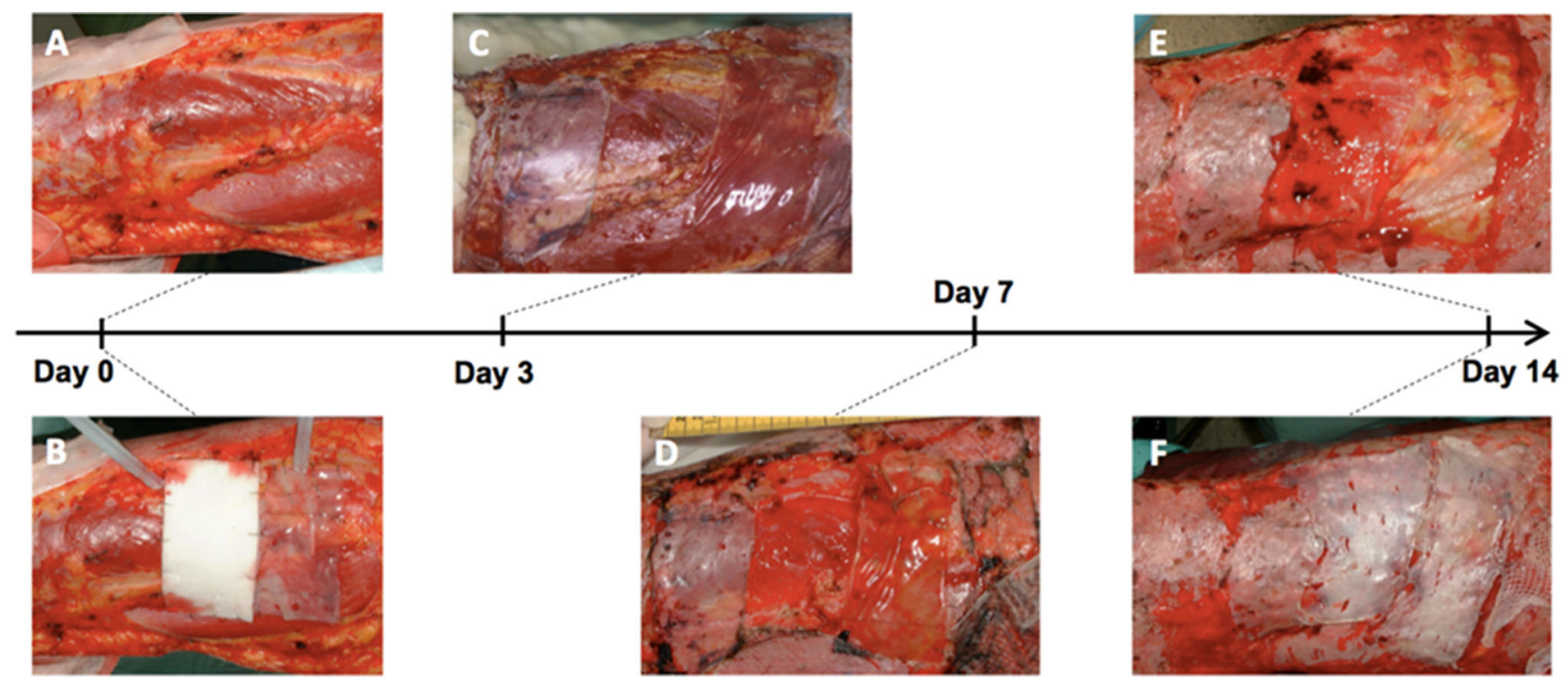

Fig. 2 Timeline of the study at days 0-14. Panel a wound bed of the test area after tangential excision. Before covering with STSG, the wound bed showed a well-vascularized muscle underneath fascia at day 0. Panel $\mathbf{b}$ one of the treatment areas was covered with a thin STSG (missing on the left side in the picture). The other two treatment areas were covered with Cellonex ${ }^{\mathrm{TM}}$ and Integra ${ }^{\circledR}$ the next
2 weeks. Panel c day 3 after operation and after removal of Cellone ${ }^{\text {TM }}$. Panel d day 7 after operation and after removal of Cellonex ${ }^{\mathrm{TM}}$. Panels e-f on day 14 after the operation the silicone layer from Integra ${ }^{\circledR}$ was removed (e) and replaced by a thin STSG (f). At that point also the Cellonex ${ }^{\mathrm{TM}}$ was removed (e) and the wound bed was covered with a thin STSG (f)

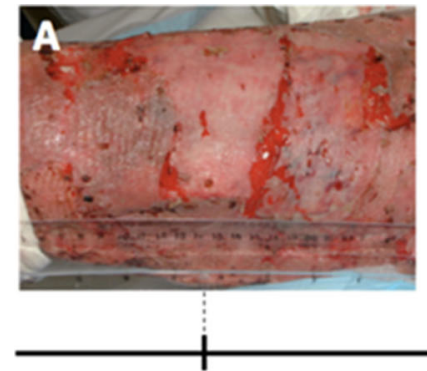

Day 21

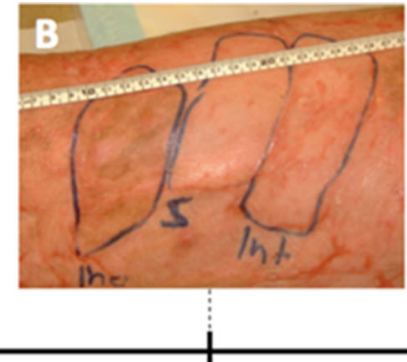

3 months

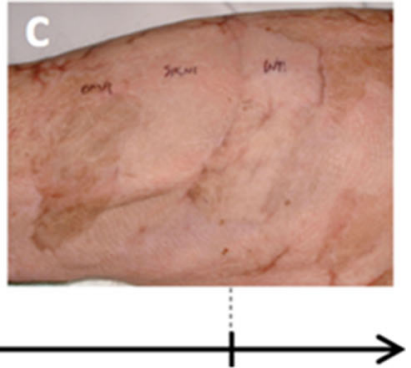

12 months
Fig. 3 Timeline of the study from day 21 to the 1-year follow-up. Panel a day 21 after the primary operation: 3 weeks after placement of STSG on treatment area without priming and 1 week after

in paraffin. Sections were stained with antibodies against CD31, MIB1 and CD163 as detailed before [12]. Serial microscopic images from the areas of the STSGs were obtained using $200 \times$ magnification. The images were analyzed using Fiji ImageJ software [15]. In Fiji ImageJ analyses, a user-defined macro was constructed to process all images in a similar manner to enable comparisons. The images were thresholded for stained pixels, and the amount of positive pixels was measured. All non-tissue areas were subtracted from the total area of the full image to gain the tissue-positive area in each image. Results are expressed as the proportion of immunostain-positive pixels divided by total positive tissue area in each image.

In morphometric analysis of $\mathrm{CD} 31^{+}$vessels, all $\mathrm{CD} 31^{+}$ circular structures in an image were manually drawn and the placement of STSG on Integra ${ }^{\circledR}$ and Cellonex ${ }^{\mathrm{TM}}$ treatment areas. Panel b three months after primary operation. Panel $\mathbf{c}$ one year after primary operation

areas of the vessels were measured with the measure function. The number of measurements was assigned as the total number of vessels per image. Vessel density was calculated by dividing the number of vessels by total tissue area in a given image.

More detailed description of the protocol is given in Supplementary information.

\section{Statistics}

Two-sided Student's $T$ test was used to detect statistically significant differences between treatment modalities at 1 week and 3 months after skin grafting. $p$ values less than 0.05 were considered significant. Linear correlation (Pearson) was calculated for $\mathrm{CD} 163^{+}$macrophages and 
$\mathrm{CD} 31^{+}$endothelial cells. Statistical calculations were done using GraphPad Prism 5.0 software.

\section{Results and discussion}

\section{Vascularity of STSGs}

Analysis of $\mathrm{CD} 31^{+}$endothelial cells and vessel structures in STSGs was performed at 1 week and 3 months after skin grafting. At 1 week, STSGs on cellulose sponge-primed sites with induced granulation tissue (GT) demonstrated the most $\mathrm{CD} 1^{+}$cells compared to STSGs both on DT and on non-primed excised wound beds (EX) (Figs. 5a-c, 6a). STSGs on EX had the least $\mathrm{CD} 31^{+}$cells, but results remained statistically insignificantly different from STSGs on DT.

In order to gain insight into vascular reactivity, we evaluated the amount of $\mathrm{CD} 1^{+}$vessels and their crosssectional areas. STSGs on induced GT had the largest vessels (Fig. 6b). Interestingly, STSGs on DT had the smallest average vessel areas of the treatment areas. Vessel density did not differ among groups, although STSGs on induced GT demonstrated a nonsignificant average increase as compared to other treatments (Fig. 6c). Taken together, these results showed that 1 week after grafting STSGs on induced GT had more $\mathrm{CD} 31^{+}$endothelial cells and larger vessels compared to STSGs on DT or on EX.

After 3 months, the initial vascular responses in STSGs on GT had returned to same level as in STSGs both on DT

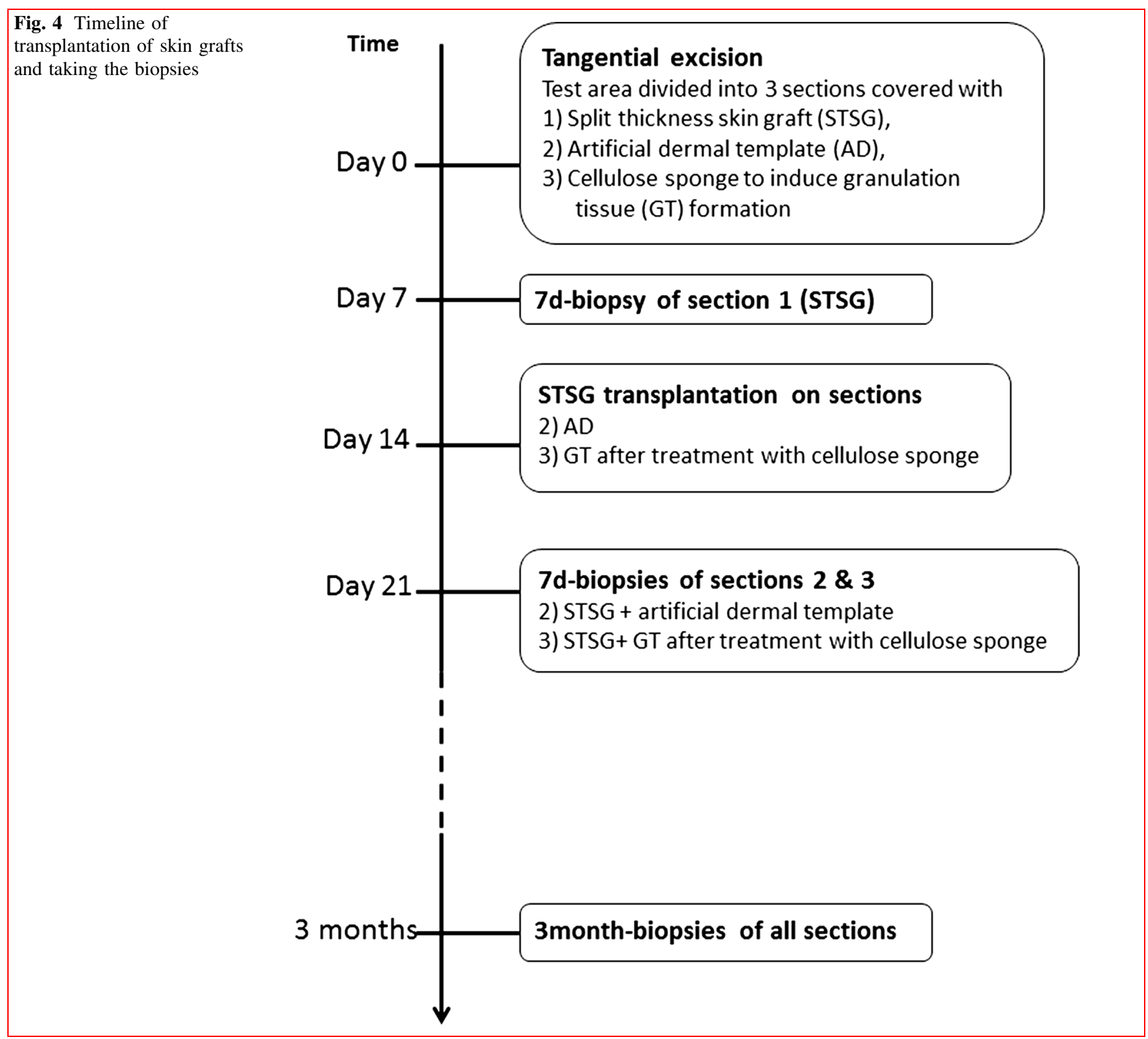




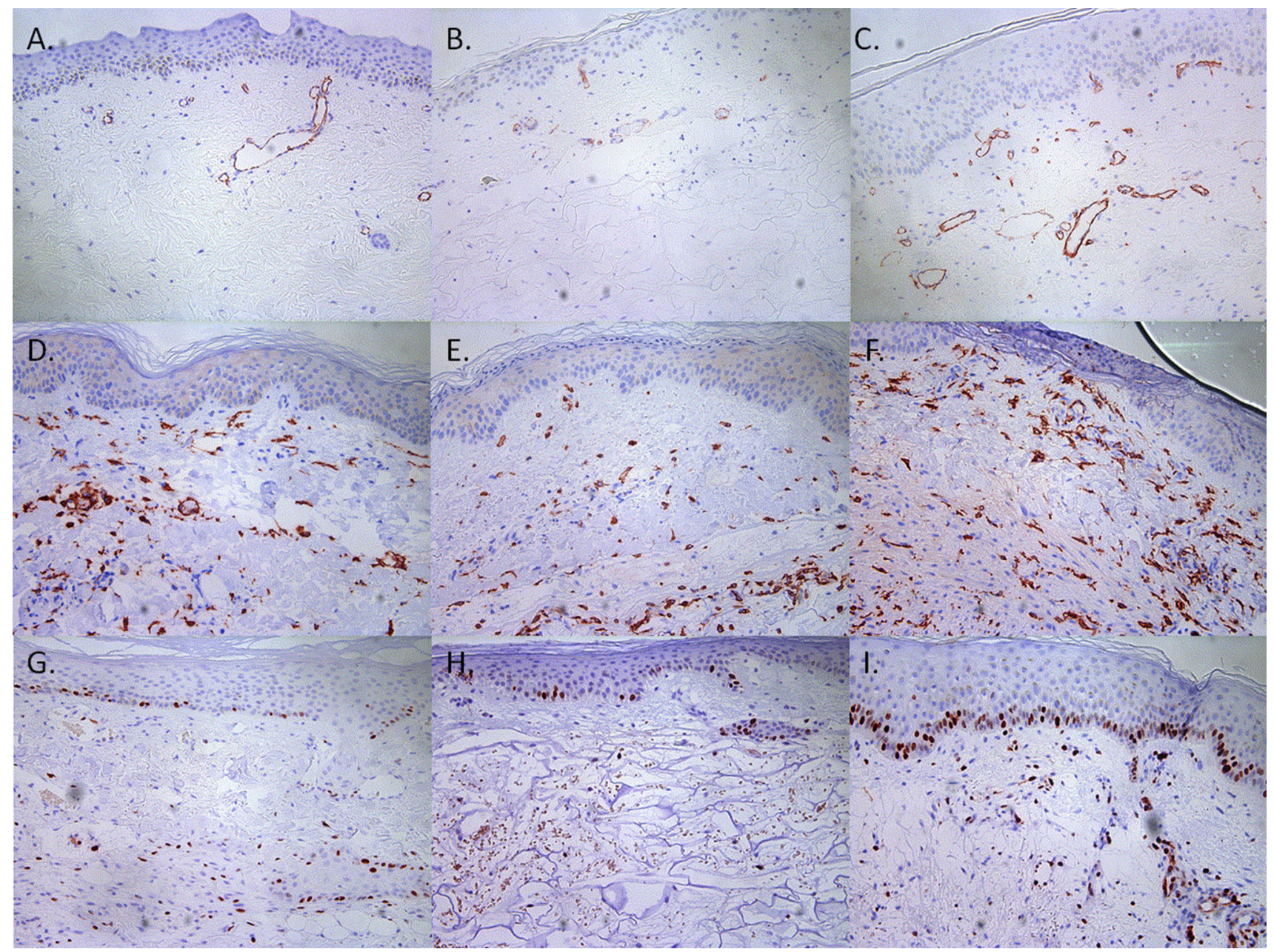

Fig. 5 Immunohistochemical stainings at 1 week after skin grafting. Panels a-c CD31, panels d-f CD163, panels g-i MIB1. Panels a, d, g are from unprimed, panels $\mathbf{b}, \mathbf{e}, \mathbf{h}$ are from Integra-primed, $\mathbf{c}, \mathbf{f}, \mathbf{i}$ are from Cellonex-primed STSGs

and on EX, suggesting that the angioactive response was acute and transient (Supplementary Fig. 1).

\section{Monocyte-macrophages in STSGs}

We utilized the CD163 macrophage marker for assessment of macrophage involvement in and infiltration into STSGs in response to wound priming. CD163 expression is associated with an anti-inflammatory repair macrophage M2 phenotype [16-18]. We found that at 1 week after graft transplantation STSGs on induced GT showed increased expression of CD163 compared to STSGs on DT and on EX (Figs. 5d-f, 7a). Amount of $\mathrm{CD}_{163^{+}}$macrophages correlated significantly with expression of CD31 in all samples (Supplementary Fig. 2), suggesting that macrophages may play a role in the vascular response of STSGs.

\section{Cell proliferation in STSGs}

Cell proliferation was evaluated by positive nuclear staining to MIB1 antibody recognizing Ki-67 antigen [19]. At 1 week, STSGs on induced GT showed the highest number of $\mathrm{MIB}^{+}$cells, whereas MIB1 staining of STSGs on EX was the lowest. Also the most proliferating cells in the STSGs' epidermal compartment were detected in samples with induced GT (Figs. 5g-i, 7b). Although cells in the STSGs' dermal compartment proliferated less than in the corresponding epidermis, a significant difference was found in dermal cell proliferation of STSGs on induced GT compared to STSGs on EX (Fig. 7c).

At 3-month time point after grafting, cellular proliferation in epidermis of STSGs decreased, and differences observed after 1 week were absent (Fig. 8a). Dermal compartment of STSGs on EX, however, now demonstrated significantly higher numbers of proliferating cells as compared to sites with pre-grafting priming (Figs. 8b, 9). 


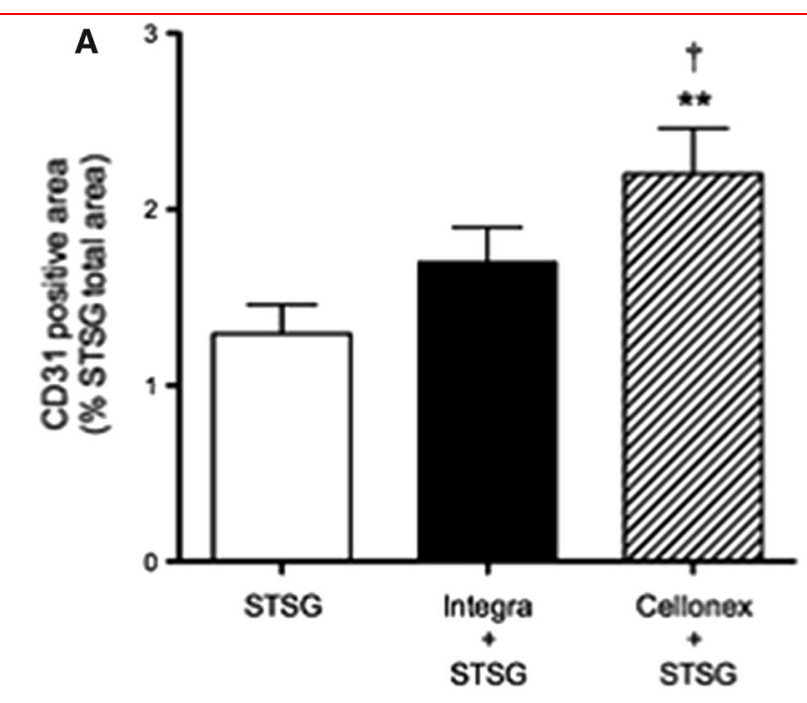

B

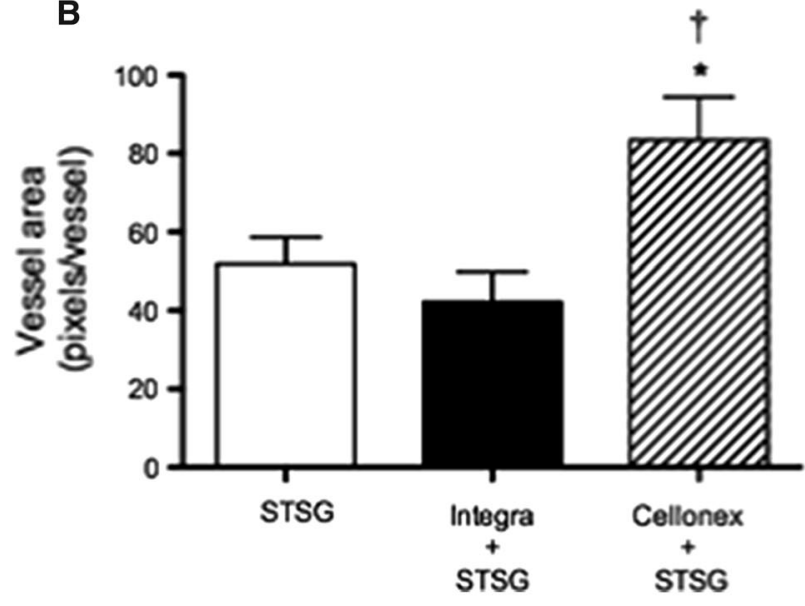

C

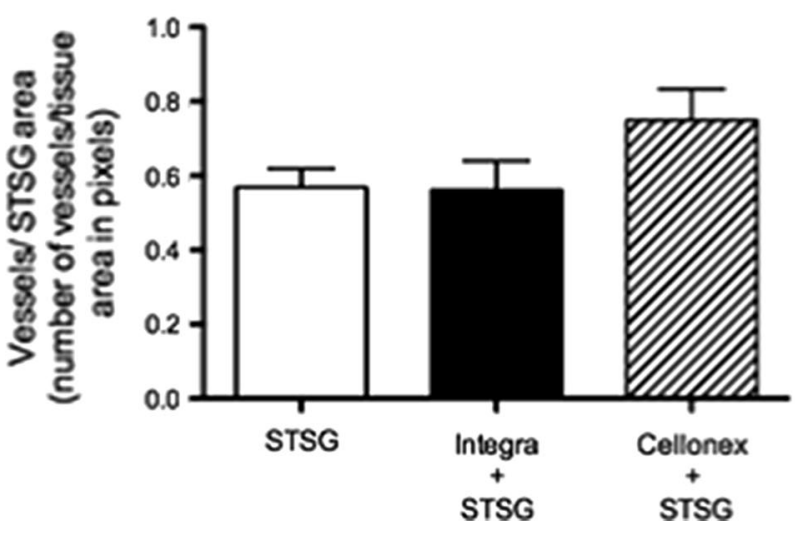

4Fig. 6 CD31 staining of endothelial cells in STSGs 1 week after transplantation a $\mathrm{CD} 31^{+}$staining area counted as percentage of the total STSG area. **represents $p<0.01$ of STSG on EX versus STSG on GT, †represents $p<0.05$ of STSG on DT versus STSG on GT, b the average size of vessels was counted from the total area inside the $\mathrm{CD} 31^{+}$staining circular structures in pixels by dividing it by the number of identified circular vessel structures. *represents $p<0.05$ of STSG on EX versus STSG on GT, †represents $p<0.05$ of STSG on DT versus STSG on GT, $\mathbf{c}$ the density of vessels (number of vessels/ tissue area in pixels)

\section{Discussion}

Although wound bed inflammation, infection and vascularity are clinically recognized predictors of graft take and integration, not much is known about the effect of wound bed priming on the graft's well-being [20]. We present here the first study, to our knowledge, to compare the effects of three different pre-grafting priming treatments of deep burn wounds excised to fascial level in a prospective controlled study in man. The gross histological and clinical evaluations as well as 1-year cosmetic and functional outcome have been published earlier [12]. In this study, we focused on histological evaluation of the different wound bed priming treatments on autologous STSGs. We surgically excised full-thickness burn wounds of ten consecutive patients with severe burns. The excised wounds were divided into three sections: one without priming, one primed with permanent artificial dermal template and one primed with temporary cellulose sponge, and the histological responses of STSGs on these sites at 1 week and 3 months after graft placement were studied.

In samples taken at the 1-week follow-up, we found a clear difference between priming methods. STSGs on recipient sites primed with cellulose sponges inducing GT formation showed a significantly stronger vascular response (increased number of $\mathrm{CD} 31^{+}$cells and bigger average vessel diameter) than those transplanted on nonprimed or artificial dermal template-primed sites. The stronger vascular responsiveness coincided also with higher proliferation rate of epidermal cells and increased amount of $\mathrm{CD} 63^{+}$macrophages in the dermis compared to STSGs on other test sites. This reactive response was transient and had subsided at 3 months. Our results underscore the importance of wound bed priming for promoting early host-graft interactions and integration. This wound bed priming method could be useful in situations where areas of poor vascularization cannot be grafted, due to any reason. Inducing well-vascularized granulation tissue formation enables graft take avoiding unnecessary graft loss or complicated reconstruction operations. On the other hand, wound bed priming for the sake of priming alone may result in delayed grafting operation, longer stay in hospital with increased costs as well as longer period of 

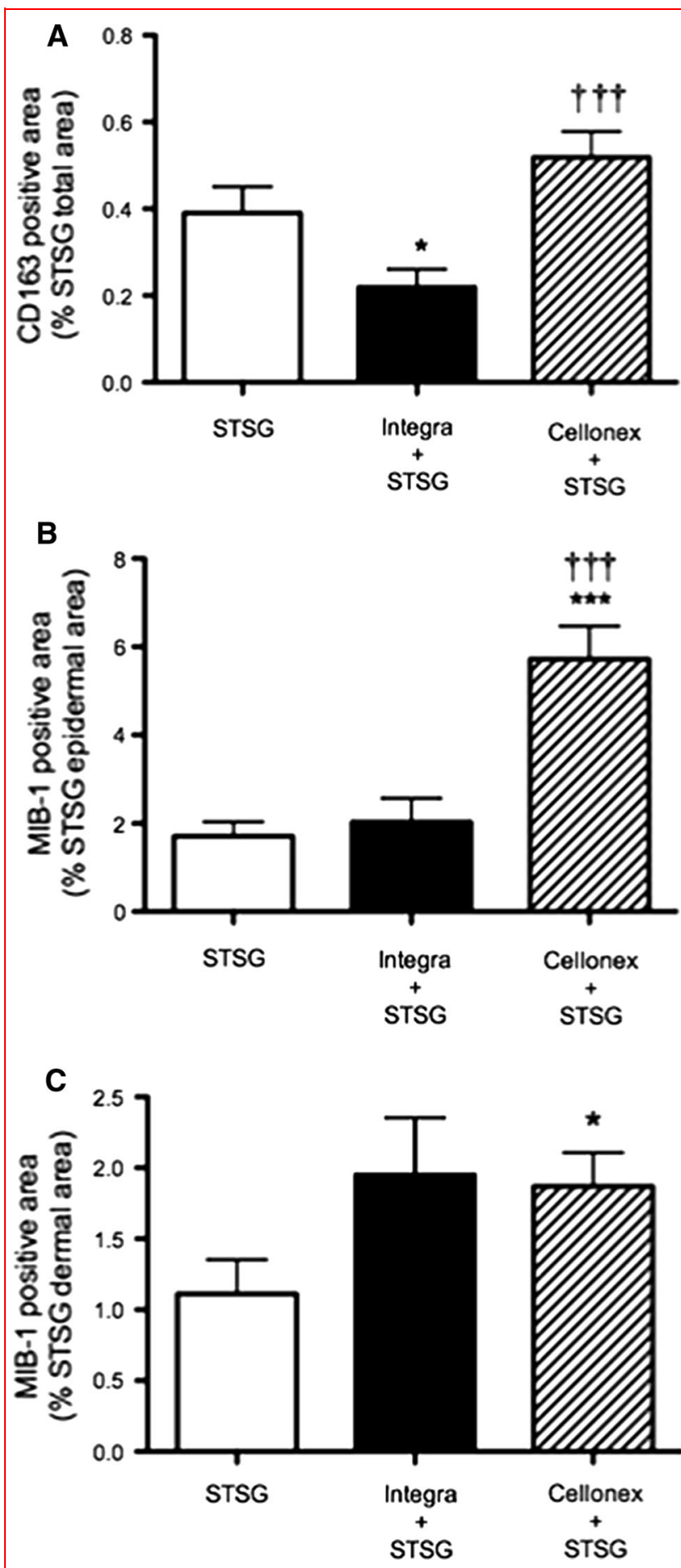

Fig. 7 One week after transplantation of STSGs on different treatment areas. a Monocyte-macrophages stained with CD163: *represents $p<0.05$ of STSG on EX versus STSG on DT, ††† represents $p<0.001$ of STSG on EX versus STSG on GT, b proliferation of epidermal cells detected by MIB1 staining, ***represents $p<0.001$ of STSG on EX versus STSG on GT and ††represents $p<0.001$ of STSG on DT versus STSG on GT, c proliferation of dermal cells measured in percentage of $\mathrm{MIB}^{+}$ staining area of the STSG's dermal area, *represents $p<0.05$ of STSG on EX versus STSG on GT
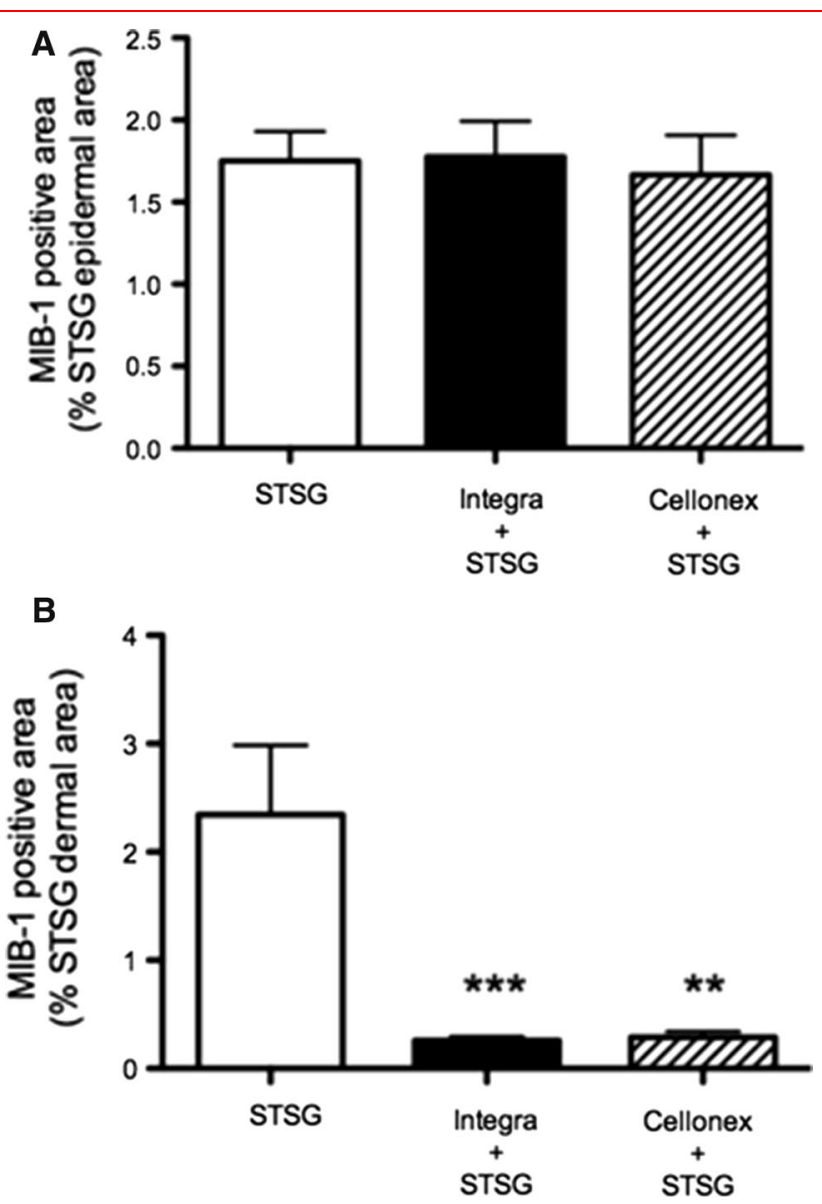

Fig. 8 Cell proliferation 3 months after operation in STSGs on different treatment areas stained with MIB1. a In the epidermis of the STSGs, $\mathbf{b}$ in the dermis of the STSGs, ***represents $p<0.001$ of STSG on EX versus STSG on DT and **represents $p<0.01$ of STSG on EX versus STSG on GT

open wounds with increased risk of infection and loss of fluids. The cellular phase of wound healing is characterized by initiation of an inflammatory response. Macrophages have been shown to play a key role in orchestrating both the quality of the inflammatory response and progression of wound healing to the proliferation phase [21]. They actively provide signals that control granulation tissue formation and maturation, stabilize vascular structures and guide myofibroblast differentiation [21]. Macrophages can differentiate into various phenotypes with distinct functions. The classically activated macrophages (M1) show proinflammatory activities, whereas alternatively activated tissue remodeling/profibrotic macrophages (M2) participate in angiogenesis, connective tissue remodeling and resolution of inflammation [21, 22]. The M2 macrophages can be further classified into subdivisions M2a, M2b, M2c and M2d according to stimuli of activation and achieved transcriptional changes [23]. The M2a and M2c subtypes, 

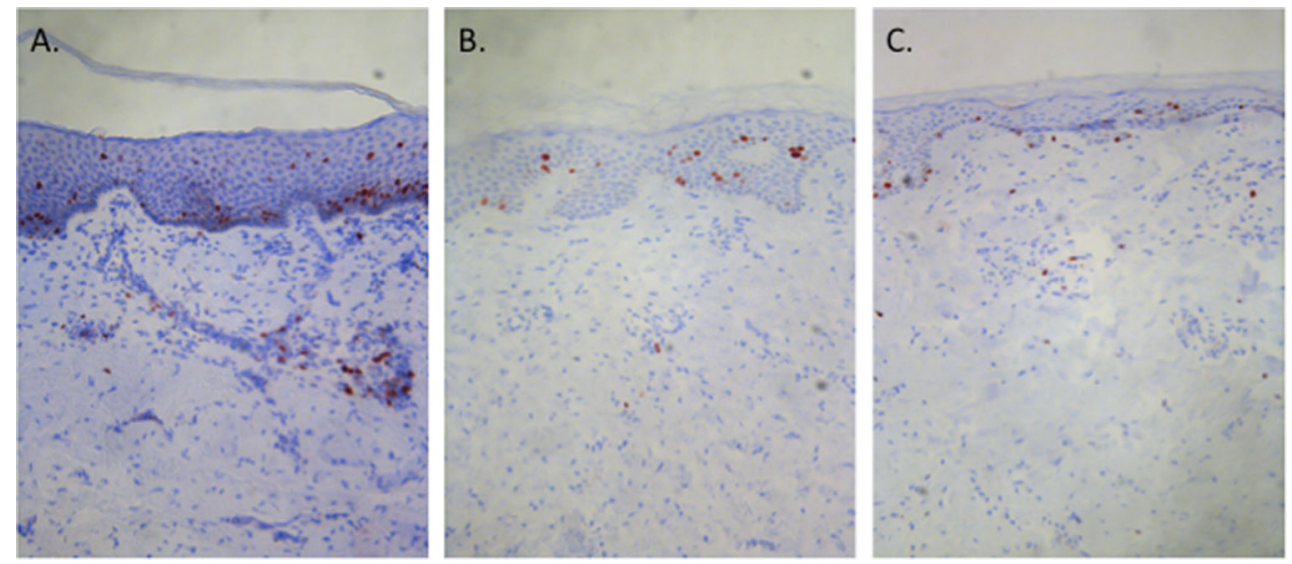

Fig. 9 Panel a STSG without priming. Panel b STSG on wound primed with Integra ${ }^{\circledR}$. Panel $\mathbf{c}$ STSG on wound primed with Cellonex ${ }^{\text {TM }}$

which are typically considered to promote angiogenesis and tissue regeneration, express the membrane protein type I CD163 antigen.

Our results showed that at 1 week after transplantation the STSGs on GT showed the highest numbers of $\mathrm{CD} 163^{+}$ macrophages of treatment sections. In fact, the viscose cellulose sponge used in the study to induce granulation tissue formation is known to attract inflammatory cells at site $[24,25]$. On the other hand, chondroitin sulfate, a constituent of the artificial dermal template used in this study, harbors anti-inflammatory properties and has been shown to decrease the influx of inflammatory cells in, for example, synovial inflammation [26]. In our study, the highest numbers of $\mathrm{CD}_{163}{ }^{+}$macrophages coincided and correlated with the highest $\mathrm{CD} 31^{+}$average area and also the highest numbers of $\mathrm{MIB} 1^{+}$proliferating cells in STSGs on GT of all STSGs. Taken together with previous reports in other biological systems, our results that place the $\mathrm{CD}_{163}{ }^{+}$macrophages in context with increased angiogenesis and cellular proliferation in human skin suggest a role for the M2 macrophages as active first-line modulators of the early wound environment.

New vessels allow passage for more cells of the immune system to enter the wound $[27,28]$ and contribute to the microenvironmental signaling [29, 30]. Based on animal studies, the kinetics of re- or neovascularization process of a skin graft $[6,20]$ has been described as follows: In the very early phase, graft survival depends on imbibition, fluid absorption. Thereafter, the re-vascularization of a skin graft proceeds in an ordered process: Vascular regression in the graft is followed by vascular ingrowth from the recipient wound bed, and connections between the vessels of the host site and the graft (inosculation) appear. Typical angiogenic changes, e.g., capillary widening, capillary bud formation and an increase in the capillary density, are seen both in wound bed and in skin graft [31]. We found that the priming of the wound bed before skin grafting had an effect on the size of blood vessels in STSGs 1 week after transplantation. The STSGs on viscose cellulose sponge-treated wound beds with induced GT showed more blood vessels with larger average areas compared to the STSGs on other test sites. Average area of the vessels was the lowest in STSGs on DT. Thus, both CD31 staining and the average area of blood vessels correlated with amount of CD163 ${ }^{+}$ macrophages 1 week after skin grafting. This further suggests that pre-treatment with non-permanent viscose cellulose sponge contributes to the early formation of highly vascularized granulation tissue in both the wound bed and the STSG.

In the epidermis of STSGs, the percentage of proliferating cells remained rather constant from 1 week to 3 months except in STSGs on induced GT. There, the amount of proliferating epidermal cells was about threefold compared to other STSGs 1 week after grafting, but equaled out at 3 months. This may be due to both better vascularization and more M2-type macrophages. Amount of proliferating dermal cells instead decreased massively down to approximately $1 / 8$ from 1 week to 3 months in all other STSGs than ones on excised wound bed. One week after grafting, dermal cells proliferated least in STSGs on excised wound bed, but on contrary to other treatment sections the $\mathrm{MIB}^{+}{ }^{+}$staining areas in dermis kept rising till 3 months at which time point positively stained areas had doubled. Reason for this is unclear and requires further studies.

The cellular events in this study do not directly translate into clinics. In our previous study with the same patient population, we observed 1 year after the primary surgery that all study areas were clinically similar with no significant differences in Vancouver scar scale or in histological assessments. This somewhat surprising result does not mean that differences in cellular or molecular level did not 
exist. The methods to assess the functional and esthetic as well as histological outcomes were chosen to be able to compare our results with those of the other groups. Unfortunately, Vancouver scar scale is not an optimal tool, but rather subjective and histological assessments are fairly gross. How the cellular findings here affect the clinical results cannot be concluded. An interesting thing is that the study areas were on fascial level and it is possible that the findings might have been different if the grafts had been inserted more superficially, e.g., on fatty tissue. One year might also be too early time point to assess "final" outcomes since maturation of scars is still in progress.

Our group is now investigating possible differences between the treatment modalities on more detailed manner and at later time points also trying to refine the assessment of the clinical outcomes more objective ways.

\section{Conclusions}

Our results demonstrate that pre-treatment of the wound bed prior to skin grafting exerts significant early histological effects on STSGs. Pre-treatment with a cellulose sponge increased the size of vessels in STSGs as well as the numbers of M2-type macrophages and proliferation of keratinocytes, suggesting better early nourishment of skin graft as compared to other materials.

Acknowledgements This study has been supported by the Scientific Committee of the Finnish Ministry of Defence and by government subsidies for Medical Research Block Grants (EVO). We thank Prof. Sirpa Asko-Seljavaara for her support. We also thank Prof. Tom Böhling for assistance and advice in immunohistochemical stainings.

\section{Compliance with ethical standards}

Conflict of interest The authors have declared that there is no conflict of interest.

\section{References}

1. Bastidas N, Ashjian P, Sharma S (2009) Acellular dermal matrix for temporary coverage of exposed critical neurovascular structures in extremity wounds. Ann Plast Surg 62:410-413. doi:10. 1097/SAP.0b013e318184ab2f

2. Viljanto J (1972) A new method for treatment of open wounds. Ann Chir Gynaecol Fenn 61(2):94-100

3. Viljanto J, Jaaskelainen A (1973) Stimulation of granulation tissue growth in burns. Ann Chir Gynaecol Fenn 62(1):18-24

4. Jones I, Currie L, Martin R (2002) A guide to biological skin substitutes. Br J Plast Surg 55(3):185-193

5. Demarchez M, Hartmann DJ, Regnier M et al (1992) The role of fibroblasts in dermal vascularization and remodeling of reconstructed human skin after transplantation onto the nude mouse. Transplantation 54(2):317-326
6. Capla JM, Ceradini DJ, Tepper OM et al (2006) Skin graft vascularization involves precisely regulated regression and replacement of endothelial cells through both angiogenesis and vasculogenesis. Plast Reconstr Surg 117(3):836-844

7. Metcalfe AD, Ferguson MWJ (2007) Tissue engineering of replacement skin: the crossroads of biomaterials, wound healing, embryonic development, stem cells and regeneration. J R Soc Interface/R Soc 4(14):413-437. doi:10.1098/rsif.2006.0179

8. Lugo LM, Lei P, Andreadis ST (2011) Vascularization of the dermal support enhances wound re-epithelialization by in situ delivery of epidermal keratinocytes. Tissue Eng Part A 17(5-6):665-675. doi:10.1089/ten.TEA.2010.0125

9. Grey JE, Enoch S, Harding KG (2006) ABC of wound healing: wound assessment. BMJ 332:285-288

10. Gurtner GC, Werner S, Barrandon Y, Longaker MT (2008) Wound repair and regeneration. Nature 453:314-321

11. Martin P, Nunan R (2015) Cellular and molecular mechanisms of repair in acute and chronic wound healing. Br J Dermatol. doi:10. $1111 /$ bjd. 13954

12. Lagus H, Sarlomo-Rikala M, Bohling T, Vuola J (2013) Prospective study on burns treated with integra(R), a cellulose sponge and split thickness skin graft: comparative clinical and histological study-randomized controlled trial. Burns 39(8): 1577-1587

13. van der Veen VC, van der Wal MB, van Leeuwen MC et al (2010) Biological background of dermal substitutes. Burns. 36(3):305-321

14. Holund B, Junker P, Garbarsch C et al (1979) Formation of granulation tissue in subcutaneously implanted sponges in rats. A comparison between granulation tissue developed in viscose cellulose sponges (Visella) and in polyvinyl alcohol sponges (Ivalon). Acta Pathol Microbiol Scand A 87A(5):367-374

15. Schindelin J, Arganda-Carreras I, Frise E et al (2012) An opensource platform for biological-image analysis. Nat Methods 9(7):676-682

16. Jetten N, Verbruggen S, Gijbels MJ et al (2014) Anti-inflammatory M2, but not pro-inflammatory M1 macrophages promote angiogenesis in vivo. Angiogenesis 17(1):109-118. doi:10.1007/ s10456-013-9381-6

17. Spiller KL, Anfang RR, Spiller KJ et al (2014) The role of macrophage phenotype in vascularization of tissue engineering scaffolds. Biomaterials 35(15):4477-4488. doi:10.1016/j.bioma terials.2014.02.012

18. Barros MH, Hauck F, Dreyer JH et al (2013) Macrophage polarisation: an immunohistochemical approach for identifying M1 and M2 macrophages. PloS One 8(11):e80908. doi:10.1371/ journal.pone.0080908

19. Scholzen T, Gerdes J (2000) The Ki-67 protein: from the known and the unknown. J Cell Physiol 182(3):311-322

20. Lindenblatt N, Calcagni M, Contaldo C et al (2008) A new model for studying the revascularization of skin grafts in vivo: the role of angiogenesis. Plast Reconstr Surg 122(6):1669-1680

21. Lucas T, Waisman A, Ranjan R et al (2010) Differential roles of macrophages in diverse phases of skin repair. J Immunol 184(7):3964-3977

22. Wermuth PJ, Jimenez SA (2015) The significance of macrophage polarization subtypes for animal models of tissue fibrosis and human fibrotic diseases. Clin Transl Med 4:2. doi:10.1186/ s40169-015-0047-4

23. Röszer T (2015) Understanding the mysterious M2 macrophage through activation markers and effector mechanisms. Mediat Inflamm 2015:816460. doi:10.1155/2015/816460

24. Pajulo O, Viljanto J, Lönnberg B et al (1996) Viscose cellulose sponge as an implantable matrix: changes in the structure increase the production of granulation tissue. J Biomed Mater Res $32: 439-446$ 
25. Tommila M, Jokilammi A, Penttinen R et al (2013) Cellulose-a biomaterial with cell-guiding property. In: van de Ven, Godbout L (eds) Chapter 5 in cellulose-medical, pharmaceutical and electronic applications. InTech. ISBN 978-953-51-1191-7

26. Monfort J, Pelletier JP, Garcia-Giralt N et al (2008) Biochemical basis of the effect of chondroitin sulphate on osteoarthritis articular tissues. Ann Rheum Dis 67:735-740

27. Arroyo AG, Iruela-Arispe ML (2010) Extracellular matrix, inflammation, and the angiogenic response. Cardiovasc Res 86(2):226-235

28. Shaw TJ, Martin P (2009) Wound repair at a glance. J Cell Sci 122(Pt 18):3209-3213
29. Infanger M, Schmidt O, Kossmehl P et al (2004) Vascular endothelial growth factor serum level is strongly enhanced after burn injury and correlated with local and general tissue edema. Burns 30(4):305-311

30. Eming SA, Brachvogel B, Odorisio T et al (2007) Regulation of angiogenesis: wound healing as a model. Prog Histochem Cytochem 42(3): 115-170

31. Knapik A, Kornmann K, Kerl K et al (2014) In vivo evaluation of wound bed reaction and graft performance after cold skin graft storage: new targets for skin tissue engineering. J Burn Care Res 35:187-196 